\title{
The Effect of Intraoperative Dexmedetomidine on Cognitive Dysfunction After Surgery: A Updated Meta-Analysis
}

\author{
Qifan Yin \\ Hebei General Hospital \\ Jianli Li ( $\nabla$ jianlili8176@163.com) \\ Hebei General Hospital \\ Xuejiao Xun \\ Hebei General Hospital \\ Jinhua He \\ Hebei General Hospital \\ Dongdong Yu \\ Hebei General Hospital \\ Zhibin Wang \\ Hebei General Hospital \\ Junfang Rong \\ Hebei General Hospital
}

\section{Research}

Keywords: postoperative cognitive dysfunction, dexmedetomidine, MMSE score, Meta-analysis, perioperative period

Posted Date: March 15th, 2021

DOI: https://doi.org/10.21203/rs.3.rs-285627/v1

License: (c) (7) This work is licensed under a Creative Commons Attribution 4.0 International License. Read Full License 


\section{Abstract}

Background

Postoperative cognitive dysfunction (POCD) is one of the most common. Neuroprotective effects of dexmedetomidine(DEX) are reported in previous studies but evidence regarding the POCD is still unclear. In order to gain latest evidence, the present study analyzes the outcomes of randomized controlled trials(RCTs) which utilized DEX with general anaesthesia perioperatively.

Method

Four online databases (PubMed, Embase, the Cochrane Library, and CNKI) were used to find relevant RCTs to conduct systematic analysis. All studies comparing the incidence of POCD or MMSE score between the DEX group and the placebo or comparator group in patients undergoing general anaesthetic surgery were eligible for inclusion. Based on the inclusion and exclusion criteria, the studies were selected. This meta-analysis was performed using odds ratios (ORs) with $95 \%$ confidence intervals (Cls) for dichotomous data and standardized mean difference (SMD) and $95 \%$ Cls for continuous data as effective measures.

Results

In total of 21 studies were included in this meta-analysis. The results showed that the incidence of POCD in DEX group was significantly lower than the control group on the first $(\mathrm{OR}=0.36,95 \% \mathrm{Cl} 0.24-0.54)$, third ( $\mathrm{OR}=0.45,95 \% \mathrm{Cl} 0.33-0.61)$ and seventh $(\mathrm{OR}=0.40,95 \% \mathrm{Cl} 0.26-0.60)$ postoperative days; the MMSE scores in DEX group were higher than the control group on the first $(\mathrm{SMD}=1.24,95 \% \mathrm{Cl} 1.08-1.41)$, third $(\mathrm{SMD}=1.09,95 \% \mathrm{Cl} 0.94-1.24)$ and seventh $(\mathrm{SMD}=3.28$, $95 \% \mathrm{Cl} 1.51-5.04)$ postoperative days.

Conclusions

Intraoperative DEX use can ameliorate the POCD of patients who received surgical operations under general anesthesia, and effectively reduce the incidence of POCD and improve MMSE score.

\section{Introduction}

Postoperative cognitive dysfunction (POCD) is one of the most common complications affecting the central nervous system after general anaesthesia and surgery, especially in elderly patients, which is characterized by short-term cognitive decline and includes memory, mood, confusion, and sleep disorders(1). Its clinical manifestations include cognitive dysfunction, personality change, and memory loss, mental disorders, and social impairment(2). It may cause loss of self-care ability and Alzheimer's disease in serious case(3). Because of the long anesthesia duration and the severe surgical stress, the risk of POCD in elderly patients is incredibly increased (4). The prevalence of POCD is ranging from $10 \%$ to $60 \%$ and varies with clinical, demographic, and surgical variables, as well as the interval between surgery and assessment in older patients $(5 ; 6)$. POCD often leads to prolonged hospitalization, increased medical costs, lower likelihood of return to independent living, and increased mortality(7-9). The elderly are vulnerable to cognitive dysfunction following operation(10-12). The Mini Mental State Examination (MMSE) is commonly used to assess cognitive function postoperatively(13). However, the exact mechanism of developing POCD remains unknown(14). The majority of researchers consider that neuroinflammation exerts an crucial role in the development of POCD(15-17).

Dexmedetomidine (DEX) is a effective and highly selective a2-adrenergic receptor agonist and acts as a multifunctional drug in the treatment of various human diseases $(18 ; 19)$. A previous study has suggested that DEX is efficient in the treatment of nerve diseases through the beneficial effects of decreasing central nervous system sympathetic outflow and providing sedation and analgesia(20).DEX treatment may improve behavioral disturbances, including aggression, agitation and cognitive dysfunction(21). In addition, some clinical studies have indicated that DEX has analgesic, anxiolytic and anti-delirium effects without respiratory depression $(18 ; 22)$. These properties make it anappropriate option for sedation in the intensive care unit and in perioperative period.

Several basic researches with animal models have reported that DEX provide neuroprotective effects and improve cognitive function following surgery(23; 24).In clinical studies, many have indicated beneficial effects of DEX in improving postoperative cognitive impairment while others could not observe the similar results. Therefore, it is still unclear that whether the administration of intraoperative DEX can ameliorate POCD. In order to gain latest evidence, the present study systematically and comprehensively analyzes the outcomes of RCTs which examined neurocognitive performance and MMSE scores to investigate the effects of DEX on POCD in patients after general anaesthesia.

\section{Materials And Methods}

\section{Search strategy}

Two review authors independently searched PubMed, Embase, the Cochrane Library, China Academic Journals full-text database (CNKI) to find relevant RCTs without any language restrictions. All studies comparing the incidence of POCD or MMSE score between the DEX group and the placebo or comparator group in patients undergoing general anaesthetic surgery were eligible for inclusion. The search was updated to Dec 31,2020. The main search terms included: ("dexmedetomidine" OR “Dex") AND ("postoperative cognitive dysfunction" OR "POCD" OR "postoperative cognitive impairment"). The reference list was also checked for relevant articles.

Inclusion and exclusion criteria 
Studies were included according to the following criteria: 1) all of the enrolled studies were randomized controlled trials (RCTs); 2) adult patients undergoing surgery under general anaesthesia; 3 ) the experimental group received a single or continuously-administered intravenous dose of intraoperative DEX; the control groups received an intravenous injection of placebo or comparator; 4) the main outcomes in enrolled studies were the incidence of POCD, postoperative MMSE score; 5) all of included studies contained original data sufficient for meta-analysis.

The exclusion criteria were: 1) abstracts, letters, case reports, reviews, duplicates or nonclinical studies; 2) studied patients who underwent local or spinalepidural anesthesia; 3 ) patients administered DEX compared with other sedative agents (benzodiazepines, midazolam, and propofol) were excluded; 4) articles with poor quality, Jadad score $\leq 3$ points, were excluded; 5) studies with incomplete information or data, and articles for which we could not obtain the full text.

\section{Data extraction and quality assessment}

Two researchers independently screened the literature, extracted data and evaluated the methodologic quality of the studies identified. If disagreement occurred, a consensus was reached after discussion with a third author. All the corresponding data including the name of first author, publication year, country, age, number of participants, type of surgery, administrations for patients, incidences of POCD, MMSE score were extracted from each selected study. We evaluated methodologic quality of the RCTs enrolled using a Jadad scale. Evaluation included randomization, allocation concealment, and blinding of implementers and participants. Studies awarded with greater than three scores were considered to be of acceptable quality. The information and data of enrolled studies were entered in a standard data extraction form. Table 1 shows the extracted contents and Jadad scores.

\section{Statistical analysis}

We used Review Manager 5.3 to conduct the meta-analysis. For dichotomous data, odds ratios (ORs) with $95 \%$ confidence intervals (Cls) were used to express effect-size, while standardized mean difference (SMD) and 95\% Cls were used for continuous data. First, we conducted a heterogeneity test on included studies using the $\mathrm{I}^{2}$ test. A fixed effects model was performed to conduct the meta-analysis if no heterogeneity $\left(P>0.1\right.$ or $\left.\mathrm{I}^{2} \varangle 50.0 \%\right)$ was found among the studies. If significant heterogeneity $\left(P<0.1\right.$ or $\left.I^{2} \geq 50.0 \%\right)$ was found, a random effects model was applied for the meta-analysis. Bias or publication bias was evaluated as quality using funnel plots, Egger's and Begg's tests. A value of $P<0.05$ was considered statistically significant.

\section{Results}

\section{Study selection}

In the initial search, a total of 1,527 potential articles were identified. After the initial screening of abstracts and titles, 1,448 articles were excluded based on the inclusion and exclusion criteria. The remaining 78 articles were collected for full text review, after the secondary screening and carefully inspection of these articles, 21 studies including 2,902 patients published between 2012 and 2020 were eventually selected for final analysis. Another 57 articles were excluded; 14 studies reported animal trials or basic experiments, 13 studies demonstrated DEX combined with other sedative agents (benzodiazepines, midazolam, and propofol), 8 studies described POCD in patients with local or spinal anesthesia, the quality of 8 studies with Jadad score $\leq 3$ points were poor, 5 articles described POCD in adolescents, 5 studies did not provide complete data, 4 studies were reviews and meta-analyses. The detail processes of study screening and selection were shown in the flow diagram (Fig.1).

\section{Study characteristics}

All of the studies enrolled for our meta-analysis were RCTs. In total of 21 studies involved 2,902 patients administered DEX(1,350) and saline/comparator(1,552). All included studies published between 2012 and 2020 . Among them, 19 studies were from China, one study was performed in The United States and Africa, respectively. With regard to the incidence of POCD in our meta-analysis, 14 studies with 1,639 patients demonstrated the relationship between DEX and the incidence of POCD on the first day after surgery. 9 articles with 1,328 patients illustrated the association between DEX and the incidence of POCD on the third day after surgery. 5 literatures with 968 patients shown the relation between DEX and the incidence of POCD on the seventh day after surgery. Concerning the relationship between DEX and MMSE score, 9 studies with 800 patients described the relationship between DEX and the MMSE score on the first day after surgery. 8 articles with 976 patients reported the association between DEX and the MMSE score on the third day after surgery. 4 researches with 628 patients reported the association between DEX and the MMSE score on the seventh day after surgery. Dosage of DEX was in the range of 0.5 to $1.5 \mathrm{ug} / \mathrm{kg}$ followed by continuous infusion at a rate of 0.15 to $0.80 \mathrm{ug} / \mathrm{kg} / \mathrm{h}$. The majority of included studies investigated the elderly. The basic characteristics of each of the enrolled studies, including publication year, country, average age, number of cases, surgical type, administrations for patients, incidence of POCD, MMSE and Jadad score, were shown in Table 1. Quality of the included studies was generally moderate to good.

\section{The association between DEX and the incidence of POCD}

14 studies including 1,639 patients reported the relationship between DEX and the incidence of POCD on the first day after surgery. There was an obvious heterogeneity between the studies $\left(P=0.009, I^{2}=52 \%\right.$, Fig. 2), therefore, a random effect model was applied. Our results clearly showed that the incidence of POCD in DEX group was significantly lower than the control group (OR=0.36, 95\% Cl 0.24-0.54, Fig.2). Meta-analysis of these 14 studies depicted that the administration of intraoperative DEX could reduce the incidence of POCD on the first day after surgery compared to placebo.

9 studies including 1,328 patients elaborated the association between DEX and the incidence of POCD on the third postoperative day. Considering no obvious heterogeneity between the studies $\left(I^{2}=0 \%, P=0.49\right.$, Fig.3), a fixed-effects model was adopted. The results of our meta-analysis suggested that the incidence of POCD in DEX group was significantly lower than controls ( $O R=0.45,95 \% \mathrm{Cl} 0.33-0.61$, Fig.3). Meta-analysis of these 9 studies indicated that the intraoperative DEX could obviously lower the incidence of POCD on the third day after surgery compared to control group. 
5 literatures including 968 patients reported the incidence of POCD on the seventh day after surgery. On account of the little heterogeneity $\left(I^{2}=47 \%, P=0.11\right.$, Fig.4), we applied a fixed-effects model in this study. Our result clearly shown that the incidence of POCD in DEX group was obviously lower than the saline/comparator group on the seventh postoperative day (OR=0.40,95\% $\mathrm{Cl} 0.26-0.60$,Fig.4). Meta-analysis of these 5 studies shown that the intraoperative DEX could reduce the incidence of POCD on the seventh day after surgery compared to saline.

\section{The association between DEX and the postoperative MMSE score}

9 studies with 800 participants reported the relationship between DEX and the postoperative MMSE score on the first day after surgery. As we all know, MMSE is commonly used to assess cognitive function, a higher MMSE score indicates better cognitive function for postoperative patients. There was an obvious heterogeneity between the studies ( $P \otimes 0.00001, P^{2}=96 \%$, Fig. 5) , therefore, a random effect model was applied. Our results clearly showed that the MMSE score in DEX group was higher than the control group ( $S M D=1.24,95 \% \mathrm{Cl} 1.08-1.41$, Fig.5). Meta-analysis of these 9 studies revealed that the intraoperative use of DEX could improve postoperative cognitive function on the first day after surgery compared to placebo.

8studies involving 976 patients reported the MMSE score on the third postoperative day. A random effect model was adopted for meta-analysis considering the apparent heterogeneity $\left(P \otimes 0.00001, I^{2}=98 \%\right.$,Fig.6). The results from meta-analysis suggested that MMSE score was higher on the third postoperative day in the DEX group than the control group $(S M D=1.09,95 \% \mathrm{Cl} 0.94-1.24$, Fig.6). Meta-analysis of these 8 studies clearly showed that the intraoperative use of $\mathrm{DEX}$ could improve postoperative cognitive function on the third postoperative day compared to saline.

4 articles including 628 patients reported the association between DEX and the MMSE score on the seventh day after surgery. Due to a large heterogeneity ( $P$ ] $0.00001, I^{2}=98 \%$, Fig.7) between the studies, So, a random effect model was applied. Our results clearly showed that the MMSE score in DEX group was higher than the control group (SMD=3.28, 95\% Cl 1.51-5.04, Fig.7). Meta-analysis of these 4 studies indicated that the intraoperative DEX treatment was associated with better postoperative cognitive performance in comparison with saline treated controls on the seventh day after surgery.

\section{Publication Bias}

A funnel plot of the 9 studies reporting the incidence of POCD on the third postoperative day was symmetrical, which indicated no publication bias (Figure 8), with all the studies within the $95 \% \mathrm{Cl}$ of the funnel.

\section{Discussion}

At the present study, our meta-analysis clearly showed that the intraoperative use of DEX could obviously alleviate cognitive dysfunction, lower the incidence of POCD, and improve MMSE score compared to saline treated controls on the first, third, and seventh day following surgery. We found that DEX did reduce the incidence of POCD over saline placebo. Our results similar to previous reports in which sedation with intraoperative DEX was associated with a lower incidence of POCD compared with placebo.

As the development of society and the trend of aging population aggravates, the increasing number of elderly patients are undergoing surgery, the elderly are more vulnerable to POCD. The incidence of POCD in the elderly is high, appealing to increasing interest in identifying strategies to prevent POCD. However, given its consequences, the concrete cause of POCD is still uncertain $(25 ; 26)$. It may be related to anesthesia methods, types of surgery, complications and potential cognitive differences(27). Some previous researches have reported the main clinical manifestations of POCD includes insanity, anxiety, personality changes and memory loss(2).Mild POCD may prolong hospitalization and increase the costs of care along with changes in memory, intelligence, verbal ability, personality, and sociability which may lead to patients' inability to engage with work and life activities(5). Severe POCD may cause patients to lose the ability to speak, induce personality change and Alzheimer's disease, and impair their self-care ability(3).

Although POCD is common in older patients, the concrete pathophysiologic mechanism is still poorly understood. Our meta-analysis clearly reported that DEX can significantly reduce the incidence of POCD in patients and improve postoperative MMSE score. DEX, an imidazole derivative and a dextrorotatory isomer of medetomidine, is a novel adrenal a2 receptor agonist with high selectivity. DEX acts at the locus coeruleus in the brain stem, which contains the highest concentration of a2 adrenoceptors. It can activate the $\mathrm{G}$ protein on the a2 adrenergic receptor in the brain and spinal cord, and inhibit the excretion of norepinephrine and neuronal discharge, which affects the sympathetic nervous system and exerts its sedative, anti-anxiety and analgesic effects(28; 29). There are many reasons for cognitive dysfunction in patients after surgery. Age, preoperative underlying disease, type of surgery, duration of anesthesia, postoperative infection, respiratory complications, intraoperative anesthesia, and sedation of the patients may be associated with POCD(30). An association between POCD and the inflammatory response has also been reported. Studies examined whether systemic inflammation in response to surgical trauma leads to subsequent memory impairment and hippocampal inflammation in a mouse model of orthopaedic surgery, and found that inflammation played a critical role in the pathogenesis of POCD and could be reversed by nonspecific inhibitor of inflammation $(31 ; 32)$. The trauma of surgery stimulates the immune cascade and the release of inflammatory mediators, which may then provoke POCD. DEX can activate the cholinergic anti-inflammatory pathway and downregulate inflammatory factors to exert anti-inflammatory effects(33). DEX exhibits anti-anxiety effects, reduces the inflammatory stress response, stabilizes hemodynamics, analgesia and reduces the incidence of adverse reactions as well as the use of sedative anesthetics(34; 35). DEX has been indicated to inhibit inflammation and improve the characteristics of brain metabolism in organ protection studies(34; 36$)$. The present study indicated that DEX was able to reduce the incidence of POCD, which confirmed its ability to prevent cognitive dysfunction following surgery. Moreover, a number of animal experiments have shown that DEX can lead to inflammation reduction, which may also explain how it reduces the incidence of POCD $(37$; 38$)$. Therefore, there is a certain protective effect on the brain and myocardium of patients for intraoperative DEX treatment, which significantly improves the occurrence of POCD (39). 
Overall, the previous meta-analyses published by Yang et al and Lei et al have also found that intraoperative DEX use was associated with reduction in the incidence of POCD and increased the MMSE score compared to saline group $(15 ; 40)$. However, the included articles of their studies were too few and the quality was relatively poor. This field about POCD has seen rapid advancement during the past years, and accumulating studies have emerged. It's necessary for us to perform a systematic review and meta-analysis to evaluate whether the results of recently published or updated trials have changed the previous analysis. In our meta-analysis, Intraoperative DEX use indeed obviously reduced the incidence of POCD, and improved MMSE score compared to saline group on the first, third, and seventh postoperative day.

Some limitations were associated with our study. First, the number of studies and the corresponding sample size were relatively limited, and the doses and methods of administration of DEX given to patients varied substantially. Secondly, the enrolled studies' inclusion and exclusion criteria, body weight, anesthetic doses, duration of surgery, surgical blood loss and consequently the characteristics of the patient cohorts, were also varied, which might have led to heterogeneity. Clinical heterogeneity of the participants can also influence the results of a meta-analysis. Finally, we have to admit that the result of POCD was lack of strength due to the only MMSE test. Different studies had different definition of POCD, which might lead to high heterogeneity in current study.

\section{Conclusion}

In conclusion, DEX can ameliorate the POCD of patients who received surgical operations under general anesthesia, and effectively reduce the incidence of POCD and improve MMSE score. Thus, it can serve as a kind of adjuvant drug for general anesthesia in clinical practice.

\section{Abbreviations}

POCD Postoperative cognitive dysfunction

DEX Dexmedetomidine

RCTs Randomized controlled trials

MMSE Mini Mental State Examination

ORs Odds ratios

Cls Confidence intervals

SMD Standardized mean difference

NA Not available

\section{Declarations}

Acknowledgement]

None

\section{Ethics approval and consent to participate}

Ethical approval for this study was not necessary because the study was a review of existing literature and did not involve the processing of any individual patient data.

\section{Consent for publication}

Not applicable.

\section{Availability of supporting data}

The datasets used and analyzed during the current study are available from the corresponding author on reasonable request.

\section{Conflict of Interest Statement}

The authors declare no conflicts of interest.

\section{Funding}

The study was supported by the Key Research and Development Program of Hebei Province

(19277714D).

\section{Author contributions}


Qifan Yin and Xuejiao Xun conducted the analysis, analyzed the data; Qifan Yin and Jianli Li wrote the manuscript; Jinhua He and Dongdong Yu screened the literature, extracted data; Zhibin Wang evaluated the quality of the enrolled studies; Jianli Li and Junfang Rong provided key information and critical comments. The authors of Qifan Yin and Jianli Li have made equal contributions to this manuscript, and they were all the co-first author. All authors read and approved the final manuscript.

\section{References}

1. Hood R, Budd A, Sorond FA, Hogue CW. 2018. Peri-operative neurological complications. Anaesthesia 73 Suppl 1:67-75

2. Hermanides J, Qeva E, Preckel B, Bilotta F. 2018. Perioperative hyperglycemia and neurocognitive outcome after surgery: a systematic review. Minerva Anestesiol 84:1178-88

3. Yuan SM, Lin H. 2019. Postoperative Cognitive Dysfunction after Coronary Artery Bypass Grafting. Braz J Cardiovasc Surg 34:76-84

4. Visovatti MA, Reuter-Lorenz PA, Chang AE, Northouse L, Cimprich B. 2016. Assessment of Cognitive Impairment and Complaints in Individuals With Colorectal Cancer. Oncol Nurs Forum 43:169-78

5. M“1ller A, Lachmann G, Wolf A, M?rgeli R, Weiss B, Spies C. 2016. Peri- and postoperative cognitive and consecutive functional problems of elderly patients. Curr Opin Crit Care 22:406-11

6. Needham MJ, Webb CE, Bryden DC. 2017. Postoperative cognitive dysfunction and dementia: what we need to know and do. Br J Anaesth 119:i115-i25

7. Monk TG, Weldon BC, Garvan CW, Dede DE, van der Aa MT, et al. 2008. Predictors of cognitive dysfunction after major noncardiac surgery. Anesthesiology 108:18-30

8. Steinmetz J, Christensen KB, Lund T, Lohse N, Rasmussen LS, Group I. 2009. Long-term consequences of postoperative cognitive dysfunction. Anesthesiology 110:548-55

9. Brown CH, Laflam A, Max L, Lymar D, Neufeld KJ, et al. 2016. The Impact of Delirium After Cardiac Surgical Procedures on Postoperative Resource Use. Ann Thorac Surg 101:1663-9

10. Miller D, Lewis SR, Pritchard MW, Schofield-Robinson OJ, Shelton CL, et al. 2018. Intravenous versus inhalational maintenance of anaesthesia for postoperative cognitive outcomes in el derly people undergoing non-cardiac surgery. Cochrane Database Syst Rev 8:Cd012317

11. Rundshagen I. 2014. Postoperative cognitive dysfunction. Dtsch Arztebl Int 111:119-25

12. Kelly M. 1997. Postoperative delirium in the elderly. Todays Surg Nurse 19:10-2

13. Vide S, Gamb ${ }^{2}$ S PL. 2018. Tools to screen and measure cognitive impairment after surgery and anesthesia. Presse Med 47:e65-e72

14. Benhamou D, Brouquet A. 2016. Postoperative cerebral dysfunction in the elderly: Diagnosis and prophylaxis. J Visc Surg 153:S27-s32

15. Lei D, Sha Y, Wen S, Xie S, Liu L, Han C. 2020. Dexmedetomidine May Reduce IL-6 Level and the Risk of Postoperative Cognitive Dysfunction in Patients After Surgery: A Meta-Analysis. Dose Response 18:1559325820902345

16. Li Y, He R, Chen S, Qu Y. 2015. Effect of dexmedetomidine on early postoperative cognitive dysfunction and peri-operative inflammatio $\mathrm{n}$ in elderly patients undergoing laparoscopic cholecystectomy. Exp Ther Med 10:1635-42

17. Zhang J, Liu G, Zhang F, Fang H, Zhang D, et al. 2019. Analysis of postoperative cognitive dysfunction and influencing factors of dexmedetomidine anesthesia in elderly patients with colorectal cancer. Oncol Lett 18:3058-64

18. Shutes BL, Gee SW, Sargel CL, Fink KA, Tobias JD. 2018. Dexmedetomidine as Single Continuous Sedative During Noninvasive Ventilation: Typical Usage, Hemodyna mic Effects, and Withdrawal. Pediatr Crit Care Med 19:287-97

19. Kim KN, Lee HJ, Kim SY, Kim JY. 2017. Combined use of dexmedetomidine and propofol in monitored anesthesia care: a randomized controlled st udy. BMC Anesthesiol 17:34

20. Wang X, Zhao B, Li X. 2015. Dexmedetomidine attenuates isoflurane-induced cognitive impairment through antioxidant, anti-inflamma tory and antiapoptosis in aging rat. Int $J$ Clin Exp Med 8:17281-8

21. Prommer E. 2011. Review article: dexmedetomidine: does it have potential in palliative medicine? Am J Hosp Palliat Care 28:276-83

22. Ohtsuka M. 2012. [Dexmedetomidine for postoperative sedation in elderly patients with cognitive impairment]. Masui 61:379-83

23. Wu J, Vogel T, Gao X, Lin B, Kulwin C, Chen J. 2018. Neuroprotective effect of dexmedetomidine in a murine model of traumatic brain injury. Sci Rep 8:4935

24. Hu J, Vacas S, Feng X, Lutrin D, Uchida Y, et al. 2018. Dexmedetomidine Prevents Cognitive Decline by Enhancing Resolution of High Mobility Group Box 1 Prote in-induced Inflammation through a Vagomimetic Action in Mice. Anesthesiology 128:921-31

25. Bilotta F, Qeva E, Matot I. 2016. Anesthesia and cognitive disorders: a systematic review of the clinical evidence. Expert Rev Neurother 16:1311-20

26. Brioni JD, Varughese S, Ahmed R, Bein B. 2017. A clinical review of inhalation anesthesia with sevoflurane: from early research to emerging topics. $J$ Anesth 31:764-78

27. Kunnimalaiyaan S, Sokolowski KM, Balamurugan M, Gamblin TC, Kunnimalaiyaan M. 2015. Xanthohumol inhibits Notch signaling and induces apoptosis in hepatocellular carcinoma. PLoS One 10:e0127464

28. Hoy SM, Keating GM. 2011. Dexmedetomidine: a review of its use for sedation in mechanically ventilated patients in an intensive care setting and for procedural sedation. Drugs 71:1481-501

29. Mantz J, Josserand J, Hamada S. 2011. Dexmedetomidine: new insights. Eur J Anaesthesio/ 28:3-6

30. 2005. [Postoperative cognitive dysfunction.]. Rev Bras Anestesio/ 55:481-2

Page 6/11 
31. Zhu YJ, Peng K, Meng XW, Ji FH. 2016. Attenuation of neuroinflammation by dexmedetomidine is associated with activation of a cholinergic an tiinflammatory pathway in a rat tibial fracture model. Brain Res 1644:1-8

32. Su ZY, Ye Q, Liu XB, Chen YZ, Zhan H, Xu SY. 2017. Dexmedetomidine mitigates isoflurane-induced neurodegeneration in fetal rats during the second trimes ter of pregnancy. Neural Regen Res 12:1329-37

33. Pavlov VA, Tracey KJ. 2005. The cholinergic anti-inflammatory pathway. Brain Behav Immun 19:493-9

34. Lee SH, Kim N, Lee CY, Ban MG, Oh YJ. 2016. Effects of dexmedetomidine on oxygenation and lung mechanics in patients with moderate chronic obstru ctive pulmonary disease undergoing lung cancer surgery: A randomised double-blinded trial. Eur J Anaesthesio/33:275-82

35. Li XM, Li F, Liu ZK, Shao MT. 2015. Investigation of one-lung ventilation postoperative cognitive dysfunction and regional cerebral oxyge $\mathrm{n}$ saturation relations. J Zhejiang Univ Sci B 16:1042-8

36. Kernan S, Rehman S, Meyer T, Bourbeau J, Caron N, Tobias JD. 2011. Effects of dexmedetomidine on oxygenation during one-lung ventilation for thoracic surgery in adults. J Minim Access Surg 7:227-31

37. Martin-Flores M, Sakai DM, Honkavaara J, Campoy L. 2018. Hemodynamic effects of low-dose atipamezole in isoflurane-anesthetized cats receiving an infusion of dexmedetomidine. J Feline Med Surg 20:571-7

38. Han XR, Wen X, Wang YJ, Wang S, Shen M, et al. 2018. MicroRNA-140-5p elevates cerebral protection of dexmedetomidine against hypoxic-ischaemic brain damag e via the Wnt/|Â-catenin signalling pathway. J Cell Mol Med 22:3167-82

39. Teegarden BM, Prough DS. 2016. Delirium: Getting Back on Track. Crit Care Med 44:1265-6

40. Yang W, Kong LS, Zhu XX, Wang RX, Liu Y, Chen LR. 2019. Effect of dexmedetomidine on postoperative cognitive dysfunction and inflammation in patients after $\mathrm{g}$ eneral anaesthesia: A PRISMA-compliant systematic review and meta-analysis. Medicine (Baltimore) 98:e15383

\section{Tables}

Table 1:The basic characteristics of the enrolled studies 


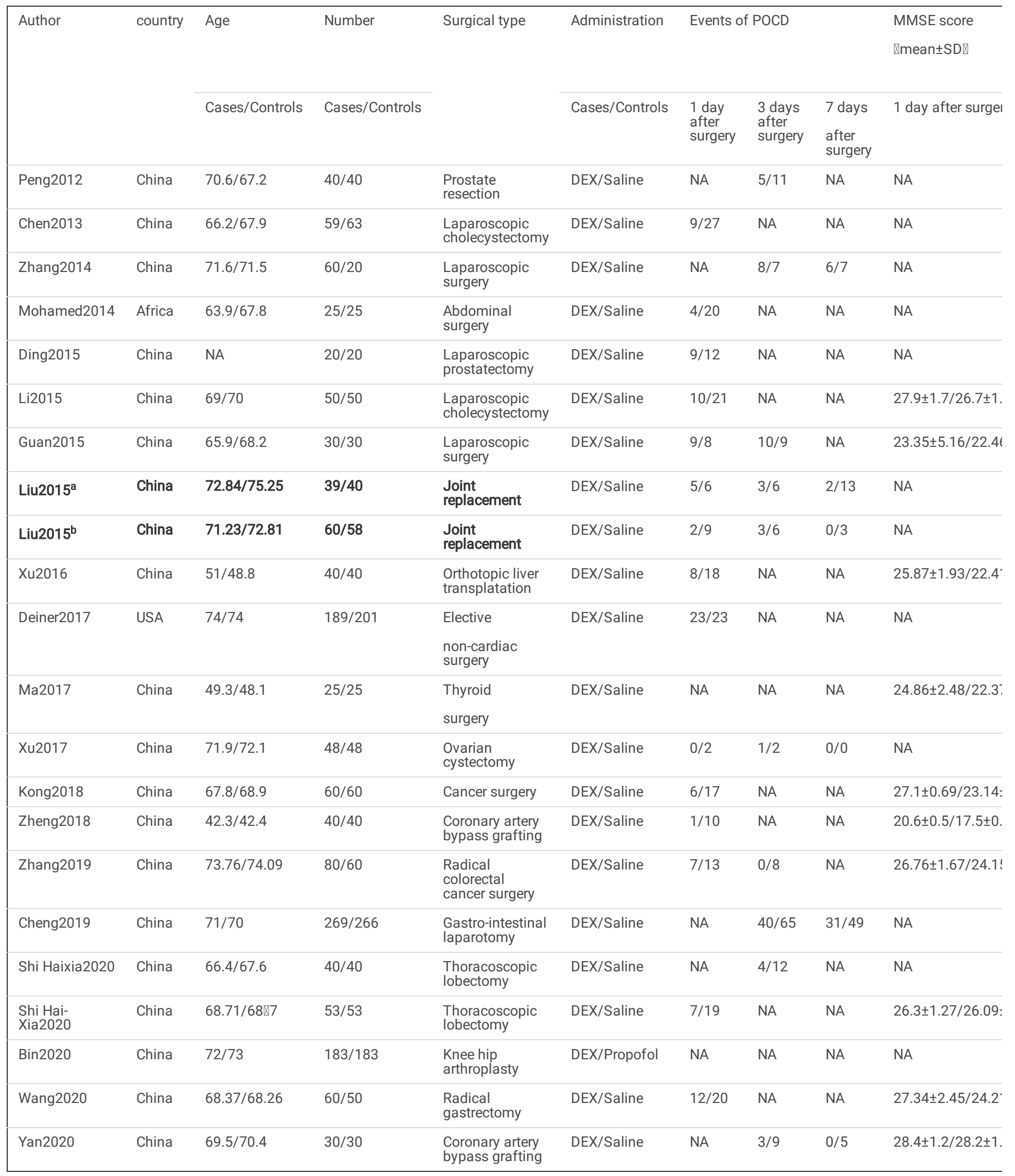

(DEX: dexmedetomidine; NA囚Not available; Liu2015a and Liu2015 ${ }^{\mathrm{b}}$ are from same study)

\section{Figures}




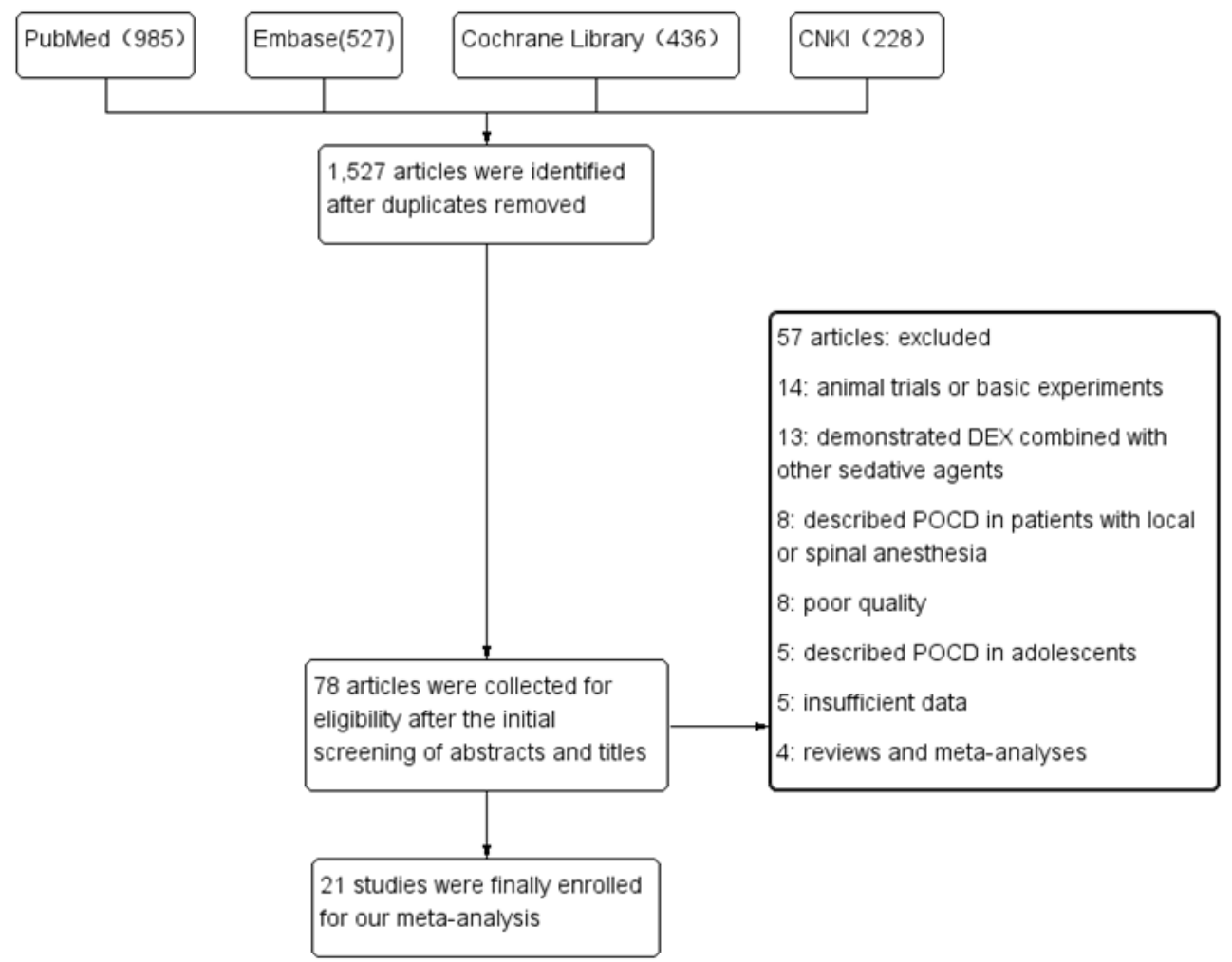

\section{Figure 1}

Flow diagram of the literature selection

$$
\text { Experimental Control }
$$

Odds Ratio

Odds Ratio

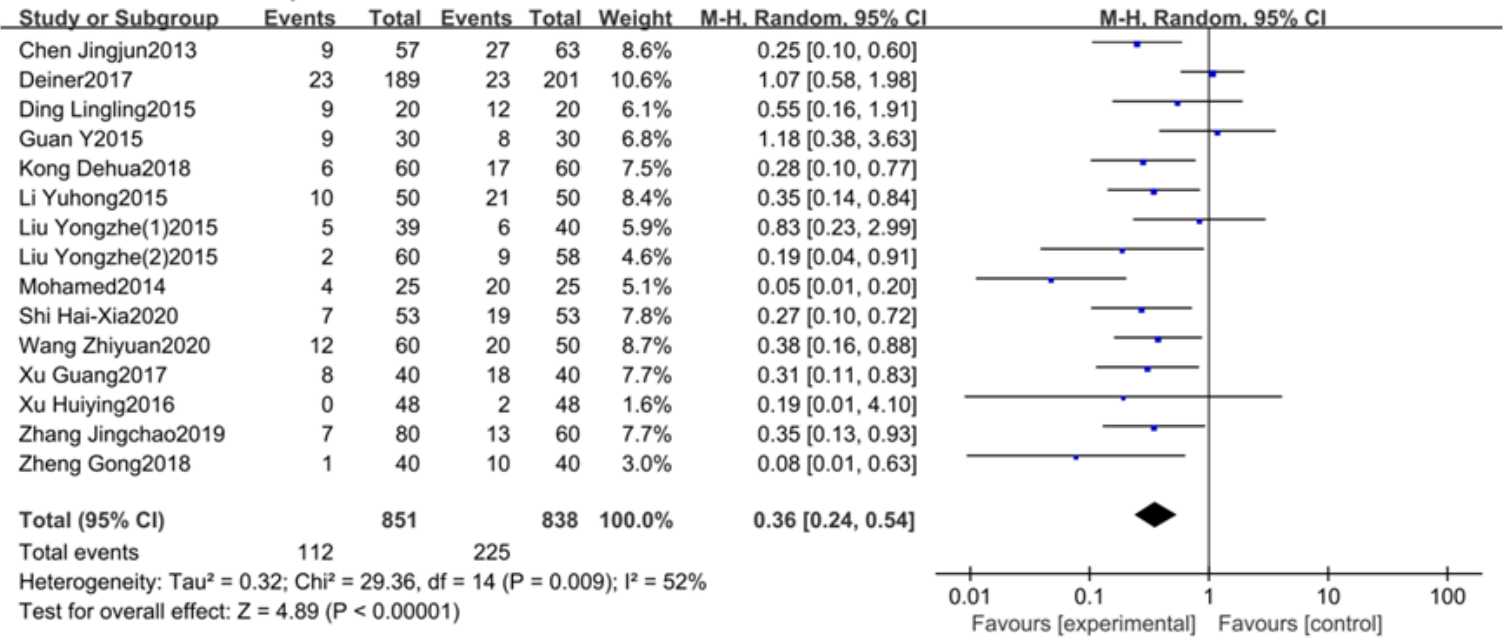

\section{Figure 2}

The association between DEX and the incidence of POCD on the first day after surgery 


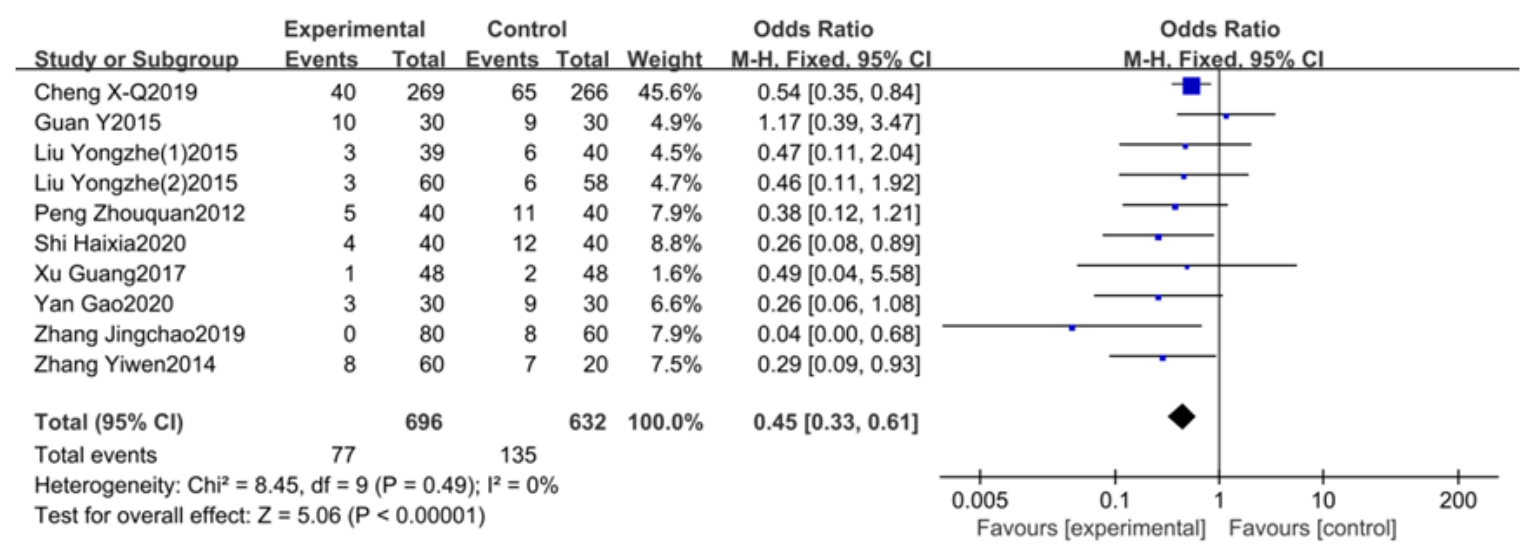

\section{Figure 3}

The association between DEX and the incidence of POCD on the third day after surgery

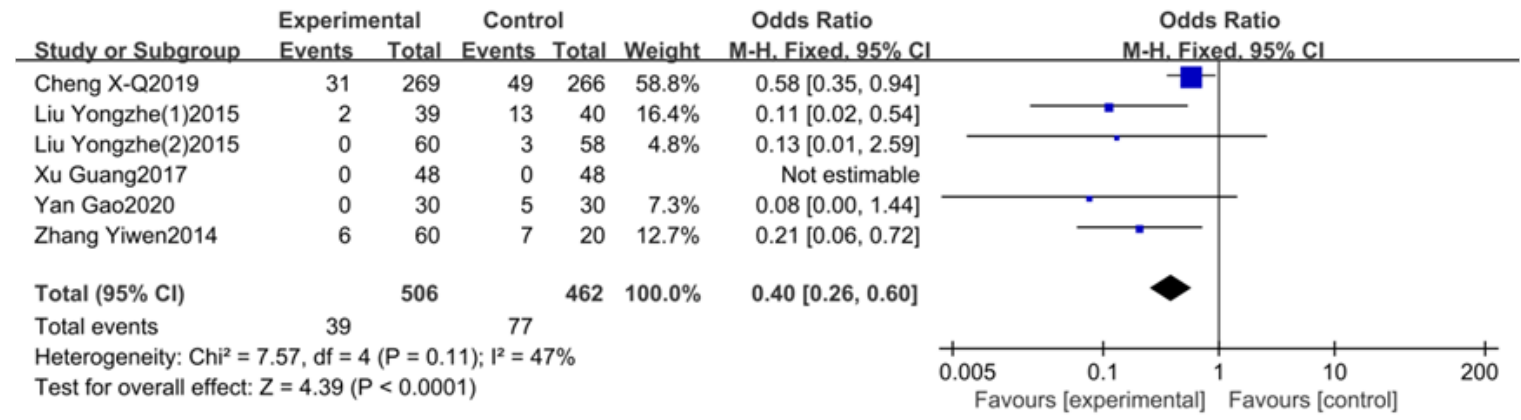

Figure 4

The association between DEX and the incidence of POCD on the seventh day after surgery

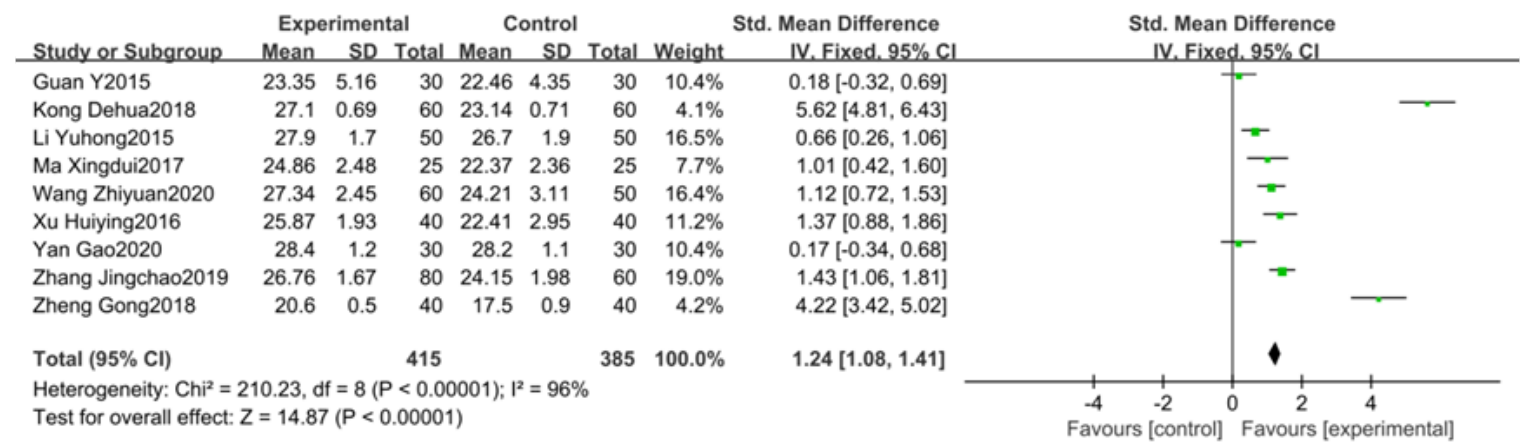

Figure 5

The association between DEX and the MMSE score on the first day after surgery

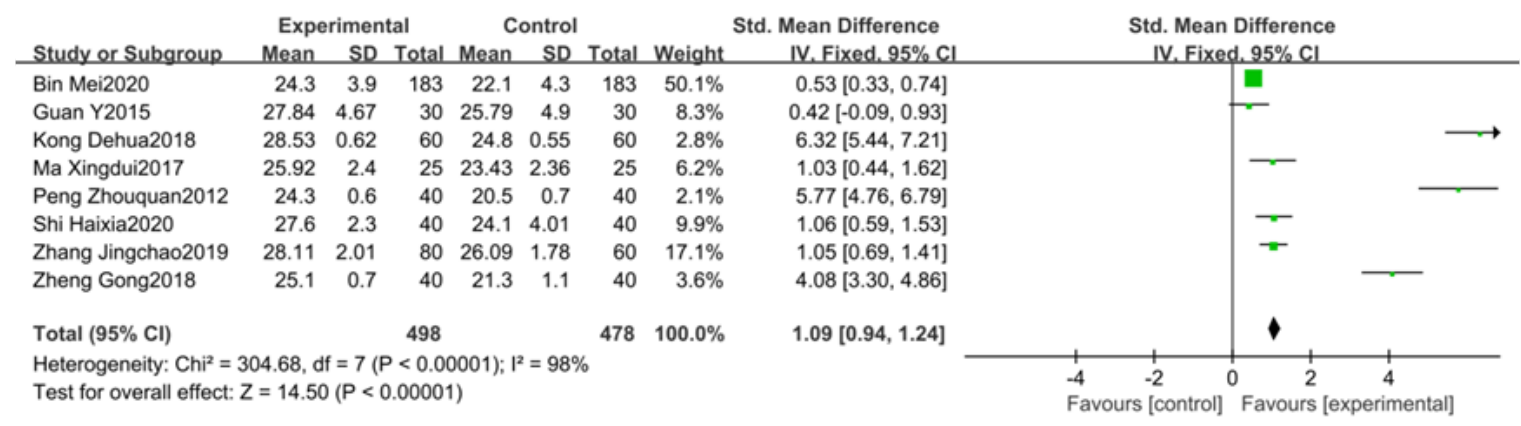

\section{Figure 6}

The association between DEX and the MMSE score on the third day after surgery 


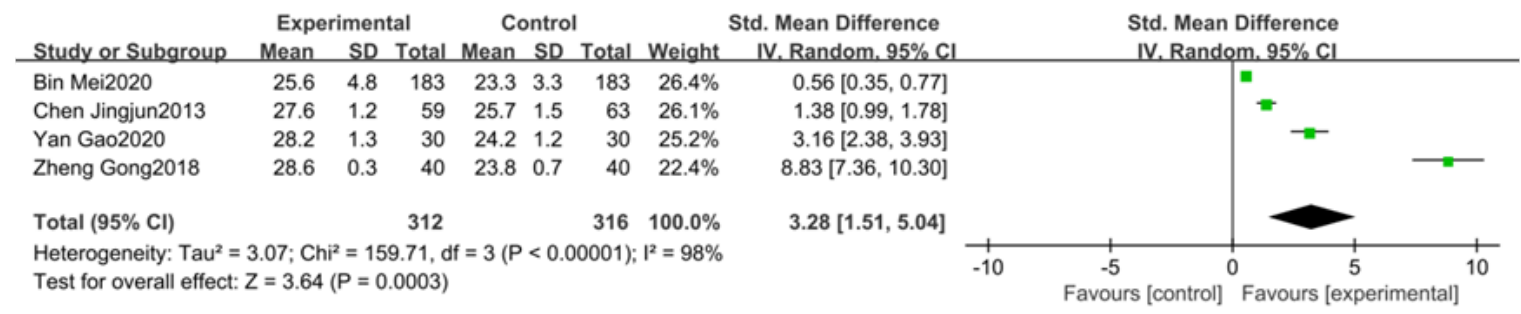

Figure 7

The association between DEX and the MMSE score on the seventh day after surgery

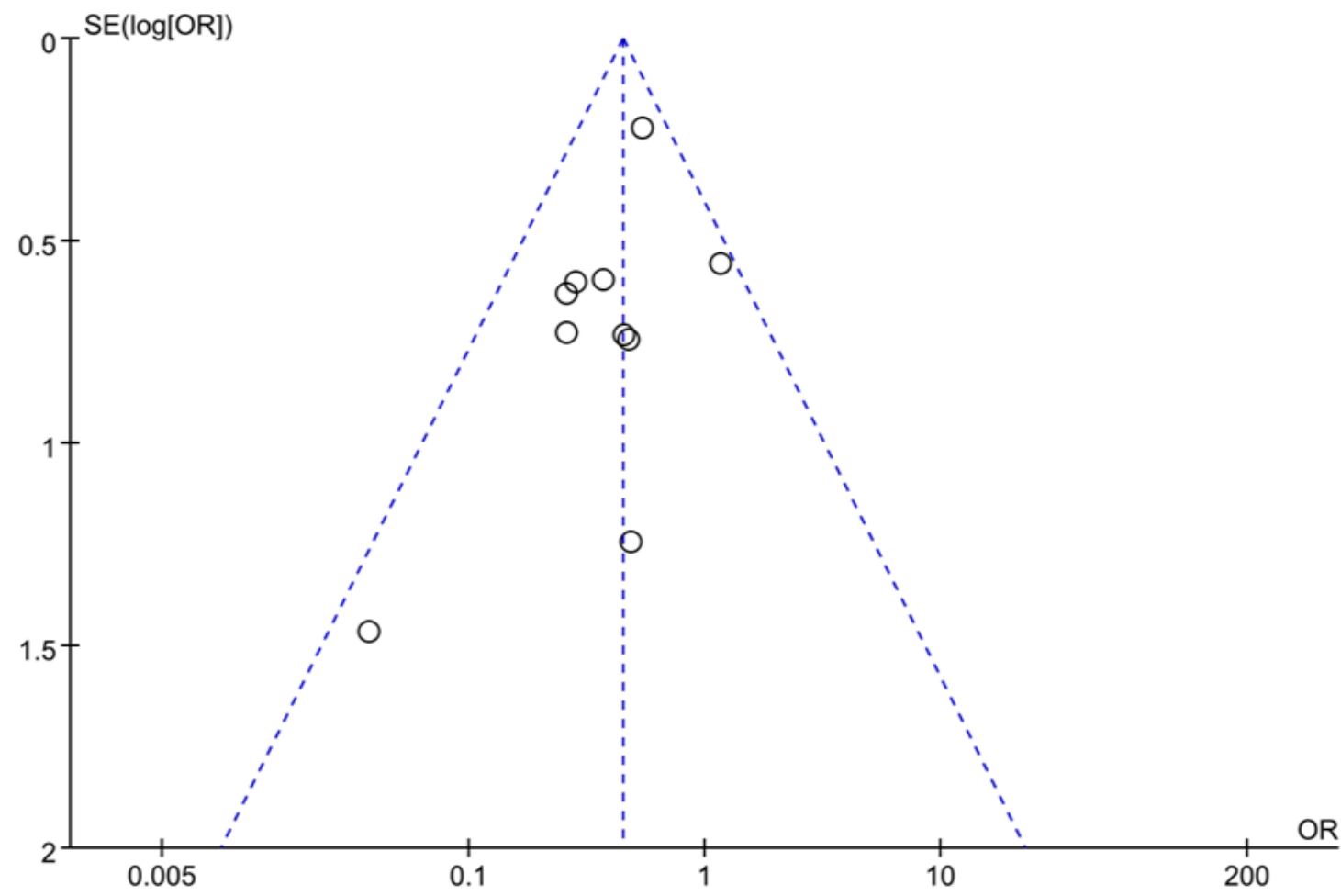

Figure 8

The funnel plot 